\title{
Fermi-Bose duality of the Dirac equation and extended real Clifford-Dirac algebra
}

\author{
I.Yu. Krivsky, V.M. Simulik* \\ Institute of Electron Physics, National Academy of Sciences of Ukraine, \\ 21 Universitetska Str., 88000 Uzhgorod, Ukraine
}

Received May 12, 2010, in final form June 29, 2010

\begin{abstract}
We have proved on the basis of the symmetry analysis of the standard Dirac equation with nonzero mass that this equation may describe not only fermions of spin $1 / 2$ but also bosons of spin 1 . The new bosonic symmetries of the Dirac equation in both the Foldy-Wouthuysen and the Pauli-Dirac representations are found. Among these symmetries (together with the 32-dimensional pure matrix algebra of invariance) the new, physically meaningful, spin 1 Poincaré symmetry of equation under consideration is proved. In order to provide the corresponding proofs, a 64-dimensional extended real Clifford-Dirac algebra is put into consideration.
\end{abstract}

Key words: spinor field, symmetry, group-theoretical analysis, supersymmetry, Foldy-Wouthuysen representation, Clifford-Dirac algebra

PACS: 11.30-z., 11.30.Cp., 11.30.j

\section{Introduction}

The main physically meaningful result of this paper is as follows. It has been shown that the Dirac equation (with nonzero mass!) can equally well describe both spin $1 / 2$ fermionic field and spin 1 bosonic field. The Dirac's well-known result (complemented by Foldy-Wouthuysen (FW) analysis and the Bargman-Wigner classification of fields), which is presented in each handbook on quantum mechanics, contains only one (fermionic) half of our consideration.

An interest in the problem of the relationship between the Dirac and Maxwell equations, which describe a spin 1 field, emerged immediately after the creation of quantum mechanics [1 11$]$. The bosonic spin 1 representation of the Poincaré group $\mathcal{P}$, the vector $(1 / 2,1 / 2)$ and the tensor-scalar $(1,0) \otimes(0,0)$ representations of the Lorentz group $\mathcal{L}$, as the groups of invariance of the massless Dirac equation, were found in our papers [12 17]. Here $\mathcal{P}$ is the universal covering of the proper ortochronous Poincaré group $\left.\mathrm{P}_{+}^{\uparrow}=\mathrm{T}(4) \times\right) \mathrm{L}_{+}^{\uparrow}$ and $\mathcal{L}=\mathrm{SL}(2, \mathrm{C})$ is that of the Lorentz group $\mathrm{L}_{+}^{\uparrow}=$ $\mathrm{SO}(1,3)$, respectively. In this paper, using the conception of the relativistic invariance of the theory, we consider only its invariance with respect to some representations of the proper ortochronous Poincaré group (the group $\mathcal{P}$ without reflections, CPT, etc.)

This paper is directly related to our previous papers [12 17]. It is the next logical step, which follows from considerations [12 17], where each symmetry of the massless Dirac equation is mapped into the corresponding symmetry of the Maxwell equations in terms of field strengths (generated by the gradient-type current), and vice versa. Let us recall that $\mathcal{P}$-symmetries of a field equation (its invariance with respect to a certain representation of the group $\mathcal{P}$ ) have the principal physical meaning: the Bargman-Wigner classification of the fields is based on the $\mathcal{P}$-symmetries of the corresponding field equations. The relationship between the massless Dirac equation and the above mentioned Maxwell equations [12 17] means, in particular, that each equation is invariant not only with respect to the fermionic $\mathcal{P}$-representation (for the $s=1 / 2$ spin doublet), but also with respect to the bosonic tensor-scalar $\mathcal{P}$-representation (with $s=1,0$ ).

Now we are able to present the similar results for the general case when the mass in the Dirac equation is nonzero.

*E-mail: vsimulik@gmail.com, sim@iep.uzhgorod.ua 
First we seek for the additional symmetries of the FW equation [18]. Then one can easily find the corresponding symmetries of a standard Dirac equation fulfilling the well-known FW transformation [18]. Thus, we find a wide set of symmetries of the Dirac equation in both FW and standard Pauli-Dirac (PD) representations. The 32-dimensional algebra $\mathrm{A}_{32}$ of invariance is found. In the FW representation, the algebra $\mathrm{A}_{32}$ is the maximal algebra of invariance in the class of pure matrix operators (without any derivatives over the space variables). The physically meaningful spin 1 symmetries are found here as subalgebras of the $A_{32}$ algebra. Finally, we have found the new hidden Poincaré symmetry of the Dirac equation and fulfilled the Bargman-Wigner analysis of corresponding $\mathcal{P}$-representation. This analysis proved that this hidden symmetry is spin 1 Poincare symmetry of the Dirac equation. On the basis of the latter assertions we come to a conclusion that the Dirac equation may also describe the spin 1 field, i.e. not only fermions of spin $1 / 2$, but also bosons of spin 1 . Thus, hereinbelow the investigation of the spin 1 symmetries of the Dirac equation with nonzero mass started in [19, 20] is being continued.

In section 2, a brief review of general assertions about Poincaré invariance and covariance of the spinor field theory is given. The ordinary handbook consideration is rewritten in terms of the so-called primary generators, which are associated with the real (not imaginary) parameters of the groups of invariance. Several tiny and hidden assumptions of group-theoretical approach to the spinor field theory are explained.

In section 3, in the canonical FW-representation, the extended real Clifford-Dirac algebra (ERCD) is put into consideration.

In section 4, the 32-dimensional maximal pure matrix algebra $\mathrm{A}_{32}$ of invariance of the $\mathrm{FW}$ equation is found.

In section 5, the spin 1 Lorentz-symmetries of the FW and Dirac equations are obtained.

In section 6, the spin 1 Poincaré-symmetry of the $\mathrm{FW}$ equation is proved.

In section 7 , the spin 1 Poincaré-symmetry of the standard Dirac equation is put into consideration.

In section 8 , a brief remix of our previous results $12-17$ for bosonic symmetries of the massless Dirac equation is presented. The specific characteristics of the case $m=0$ in comparison with nonzero mass are briefly considered. The possibility, which is open here, of generalization of all results [12 17] to the general case of nonzero mass is mentioned.

In section 9 , the brief general conclusions are given.

We use the units $\hbar=c=1$; the summation over a twice repeated index is performed.

\section{Notations, assumptions and definitions}

Here we consider the Dirac equation

$$
\left(\mathrm{i} \gamma^{\mu} \partial_{\mu}-m\right) \psi(x)=0 ; \quad \partial_{\mu} \equiv \partial / \partial x^{\mu}, \quad \mu=\overline{0,3}=0,1,2,3, \quad \psi \in \mathrm{S}(\mathrm{M}(1,3)) \times \mathrm{C}^{4} \equiv \mathrm{S}^{4,4},
$$

as an equation in the test function space $\mathrm{S}^{4,4}$ of the 4-component functions over the Minkowski space $\mathrm{M}(1,3)$. We note that the complete set $\{\psi\} \equiv \Psi$ of solutions of the equation (1) contains the generalized solutions belonging to the space $S^{4,4 *} \supset S^{4,4}$ of the Schwartz's generalized functions, $\left\{\psi^{\text {gen }}\right\} \subset S^{4,4 *}$. The mathematically well-defined consideration of this fact demands (see, e.g., the book 21] on the axiomatic approach to the field theory) the functional representation of the elements of $\psi^{\text {gen }} \subset \mathrm{S}^{4,4 *}$, which makes the consideration very complicated. Hence, we recall that the test function space $\mathrm{S}^{4,4}$ is dense in $\mathrm{S}^{4,4 *}$. It means that any element $\psi^{\text {gen }} \subset \mathrm{S}^{4,4 *}$ can be approximated (with arbitrary degree of accuracy) by an element $\psi \in \mathrm{S}^{4,4}$ from the corresponding Cauchy sequence in the space $S^{4,4}$. Therefore, here for the equation (1) we have restricted ourselves to the supposition $\psi \in \mathrm{S}^{4,4}$. Such supposition is physically verified and essentially simplifies the consideration without any loss of generality and mathematical correctness.

The invariance of equation (1) with respect to the hidden transformations will be considered. We seek for the Bose, rather than Fermi, symmetries. In order to fulfill this programme let us briefly recall the conventional relativistic invariance of equation (1). In the modern consideration, it is the invariance with respect to the universal covering $\mathcal{P} \supset \mathcal{L}=\mathrm{SL}(2, \mathrm{C})$ of the proper ortochronous 
inhomogeneous Poincaré group $\mathrm{P}_{+}^{\uparrow} \supset \mathrm{L}_{+}^{\uparrow}=\mathrm{SO}(1,3)$. For our purposes, in the space $\mathrm{S}^{4,4}$ it is convenient to rewrite the standard Fermi (spinor) representation of the group $\mathcal{P}$ in terms of the primary $\mathcal{P}$-generators $\left(p \equiv\left(p_{\mu}\right), j \equiv\left(j_{\mu \nu}\right)\right)$. By definition, these generators are associated with the real parameters $(a, \omega) \equiv\left(a^{\mu}, \omega^{\mu \nu}=-\omega^{\nu \mu}\right)$ with well-known physical meaning. The generators $\left(p_{\mu}, j_{\mu \nu}\right)$ are the orts of the real $\mathcal{P}$-algebra, i.e. the algebra over the real numbers (we use the similar symbols for groups and their algebras). The necessity of using the primary $\mathcal{P}$-generators and the real $\mathcal{P}$-algebra will be evident below in our search for additional (hidden) symmetries of the equation (1).

In arbitrary representations, the primary $\mathcal{P}$-generators $\left(p_{\mu}, j_{\mu \nu}\right)$, as the orts of the real Lie $\mathcal{P}$-algebra, satisfy the commutation relations

$$
\left[p_{\mu}, p_{\nu}\right]=0, \quad\left[p_{\mu}, j_{\rho \sigma}\right]=g_{\mu \rho} p_{\sigma}-g_{\mu \sigma} p_{\rho}, \quad\left[j_{\mu \nu}, j_{\rho \sigma}\right]=-g_{\mu \rho} j_{\nu \sigma}-g_{\rho \nu} j_{\sigma \mu}-g_{\nu \sigma} j_{\mu \rho}-g_{\sigma \mu} j_{\rho \nu},
$$

and generate a $\mathcal{P}$-representation, which is defined by an exponential series

$$
(a, \omega) \in \mathcal{P} \rightarrow \widehat{\mathrm{F}}(a, \omega)=\exp \left(a^{\mu} p_{\mu}+\frac{1}{2} \omega^{\mu \nu} j_{\mu \nu}\right) \stackrel{\inf }{=} 1+a^{\mu} p_{\mu}+\frac{1}{2} \omega^{\mu \nu} j_{\mu \nu},
$$

where symbol $\stackrel{\text { inf }}{=}$ defines "infinitesimally, i.e. in the neighborhood of the unit element of the group $\mathcal{P} "$.

The primary generators $\left(p_{\mu}, j_{\mu \nu}\right)$ for the ordinary local spinor (Fermi) $\mathcal{P}^{\mathrm{F}}$-representation in $\mathrm{S}^{4,4}$ are the following Lie operators

$$
p_{\rho}=\partial_{\rho} \equiv \partial / \partial x^{\rho}, \quad j_{\rho \sigma}=m_{\rho \sigma}+s_{\rho \sigma} \quad\left(m_{\rho \sigma} \equiv x_{\rho} \partial_{\sigma}-x_{\sigma} \partial_{\rho}, \quad s_{\rho \sigma} \equiv \frac{1}{4}\left[\gamma_{\rho}, \gamma_{\sigma}\right]\right) .
$$

The operators $p_{\mu}, j_{\mu \nu}$ from (4) commute with the Diracian $i \gamma^{\mu} \partial_{\mu}-m$. Therefore, formulae (3) with generators (4) define the local spinor (Fermi) $\mathcal{P}^{\mathrm{F}}$-representation of the group $\mathcal{P}$ in the form

$$
\psi(x) \rightarrow \psi^{\prime}(x)=\left[\widehat{\mathrm{F}}(a, \omega) \equiv \widehat{\mathrm{F}}_{1}(\omega) \widehat{\mathrm{F}}_{2}(a, \omega)\right] \psi(x) \stackrel{\inf }{=}\left(1+a^{\mu} p_{\mu}+\frac{1}{2} \omega^{\mu \nu} j_{\mu \nu}\right) \psi(x),
$$

which is the $\mathcal{P}$-group of invariance of equation (1). In formula (5) the following notations are used:

$$
\begin{aligned}
\psi, \psi^{\prime} \in \mathrm{S}^{4,4}, \quad \widehat{\mathrm{F}}_{1}(\omega) & \equiv \exp \left(\frac{1}{2} \omega^{\mu \nu} s_{\mu \nu}\right) \stackrel{\inf }{=}\left(1+\frac{1}{2} \omega^{\mu \nu} s_{\mu \nu}\right) \sim\left(\frac{1}{2}, 0\right) \otimes\left(0, \frac{1}{2}\right) \\
\widehat{\mathrm{F}}_{2}(a, \omega) \psi(x) & \equiv \exp \left(a^{\mu} p_{\mu}+\frac{1}{2} \omega^{\mu \nu} m_{\mu \nu}\right) \psi(x)=\psi\left(\Lambda^{-1}(x-a)\right) \\
& \stackrel{\inf }{=}\left(1+a^{\mu} p_{\mu}+\frac{1}{2} \omega^{\mu \nu} m_{\mu \nu}\right) \psi(x), \quad \Lambda^{-1} \in \mathrm{P}_{+}^{\uparrow} .
\end{aligned}
$$

We pay attention to the following detail of mathematical correctness of the consideration. The space $\mathrm{S}^{4,4}$ is the common domain of definitions and values both for the generators $\left(p_{\mu}, j_{\mu \nu}\right)(4)$ and for all functions from them, which we use here (in particular, for the exponential series (3) convergent in the space $S^{4,4}$ ). Further, we mark that usually the fermionic $\mathcal{P}$-transformations of the field $\psi$ are written in the form

$$
\psi(x) \rightarrow \psi^{\prime}\left(x^{\prime}\right)=\widehat{\mathrm{F}}_{1}(\omega) \psi(x), \quad x^{\prime}=\Lambda x+a, \quad \Lambda \in \mathrm{P}_{+}^{\uparrow} .
$$

However, this form (contrary to formula (5)) does not manifestly demonstrate the mathematical definition of the group of invariance of equation (1), which is given by the definition: $\mathcal{P}^{\mathrm{F}}$ is a group of invariance of equation (1), if $\mathcal{P}^{\mathrm{F}} \Psi=\Psi$; or for arbitrary solution of equation (1): if from $\psi(x) \in \Psi$ results $\widehat{\mathrm{F}}(a, \omega) \psi(x) \in \Psi \subset \mathrm{S}^{4,4}$, where $\widehat{\mathrm{F}}(a, \omega)$ is given by (5).

Let us further note that in conventional field-theoretical approach, instead of our primary Lie generators (4), the following operators are used

$$
\begin{gathered}
p_{\rho}^{\text {stand }}=\mathrm{i} p_{\rho} \equiv \mathrm{i} \partial_{\rho}, \quad j_{\rho \sigma}^{\text {stand }}=\mathrm{i} j_{\rho \sigma} \equiv m_{\rho \sigma}^{\text {stand }}+s_{\rho \sigma}^{\text {stand }} \\
\left(m_{\rho \sigma}^{\text {stand }} \equiv \mathrm{i} x_{\rho} \partial_{\sigma}-\mathrm{i} x_{\sigma} \partial_{\rho}, \quad s_{\rho \sigma}^{\text {stand }} \equiv \frac{\mathrm{i}}{4}\left[\gamma_{\rho}, \gamma_{\sigma}\right]\right) .
\end{gathered}
$$


It is evident that these generators are associated with the pure imaginary parameters $\left(-\mathrm{i} a^{\mu},-\mathrm{i} \omega^{\mu \nu}\right)$ and with corresponding complex $\mathcal{P}$-algebra. Below, as well as in our other publications on the symmetries, primary generators (4) and customary operators (9) should not be confused.

We recall that both pure matrix operators $s_{\rho \sigma}$ and pure differential operators $m_{\rho \sigma}$ from (4) satisfy the same commutation relations as $\left[j_{\mu \nu}, j_{\rho \sigma}\right]$ in $(2)$. However, the operators $s_{\rho \sigma}$ and $m_{\rho \sigma}$ (contrary to their sum $j_{\rho \sigma}$ ) are not the symmetry operators (operators of invariance) of equation (1) (operator $\widehat{q}$ is called a symmetry operator or the operator of invariance of equation (1), if equality $\widehat{q} \Psi=\Psi$ is valid, where $\Psi \equiv\{\psi\} \subset \mathrm{S}^{4,4}$ is a complete set of solutions of equation (1), see e.g. the corresponding definition in [22]). Therefore, both pure matrix $\mathcal{L}$-representation $\widehat{\mathrm{F}}_{1}(\omega)(6)$ and infinite-dimensional $\mathcal{L}$-representation

$$
\widehat{\mathrm{F}}_{2}(\omega) \psi(x) \equiv \exp \left(\frac{1}{2} \omega^{\mu \nu} m_{\mu \nu}\right) \psi(x)=\psi\left(\Lambda^{-1}(x)\right) \stackrel{\text { inf }}{=}\left(1+\frac{1}{2} \omega^{\mu \nu} m_{\mu \nu}\right) \psi(x)
$$

in $\mathrm{S}^{4,4}$ are not the groups of invariance of equation (1). As a consequence of these facts (see arbitrary conventional consideration in relevant papers, handbooks and monographs), both matrix $\mathcal{L}$-spin $s_{\rho \sigma}$ and orbital angular momentum $m_{\rho \sigma}$ (differential $\mathcal{L}$ angular momentum) do not generate the conserved in time integral constants of motion, i.e. being taken separately both spin and orbital angular momenta of the field $\psi$ are not conserved. Therefore, both pure matrix $\widehat{\mathrm{F}}_{1}(\omega)(6)$ and infinite-dimensional $\widehat{\mathrm{F}}_{2}(\omega)$ representations in $\mathrm{S}^{4,4}$ of the Lorentz group $\mathcal{L}$ are not the groups of invariance of the Dirac equation (1).

Besides the local $\mathcal{P}^{\mathrm{F}}$-representation, the so-called induced (see, e.g. 23, 24]) $\mathcal{P}^{\mathrm{F}}$-representation for the field $\psi$ is useful and meaningful. Mathematically this representation can be related to the conception of special role of the time variable $t \in(-\infty, \infty) \subset \mathrm{M}(1,3)$. Indeed, in the general consideration of the Dirac equation in the axiomatic approach, it follows from equation (1) that the Dirac field $\psi$ satisfies identically the Klein-Gordon equation

$$
\left(\partial^{\mu} \partial_{\mu}+m^{2} \equiv \partial_{0}^{2}-\triangle+m^{2}\right) \psi(x)=0, \quad \psi \in \mathrm{S}^{4,4 *}
$$

which is the equation of hyperbolic type. Hence, the generalized solutions of the Dirac equation (1) are the ordinary functions of the time variable $x^{0}=t \in(-\infty, \infty) \subset \mathrm{M}(1,3)$ (they are the generalized functions of the variables $\vec{x} \equiv\left(x^{\ell}\right) \in \mathrm{R}^{3} \subset \mathrm{M}(1,3)$ only). Therefore, due to a special role of the time variable $x^{0}=t \in\left(x^{\mu}\right)$ (in obvious analogy with nonrelativistic theory), in general consideration one can use the quantum-mechanical rigged Hilbert space

$$
\mathrm{S}^{3,4} \subset \mathrm{H}^{3,4} \subset \mathrm{S}^{3,4 *} ; \quad \mathrm{S}^{3,4} \equiv \mathrm{S}\left(\mathrm{R}^{3}\right) \times \mathrm{C}^{4}
$$

where

$$
\mathrm{H}^{3,4} \equiv \mathrm{L}_{2}\left(\mathrm{R}^{3}\right) \times \mathrm{C}^{4} \equiv\left\{f: \mathrm{R}^{3} \rightarrow \mathrm{C}^{4}, \int \mathrm{d}^{3} x|f(t, \vec{x})|^{2}<\infty\right\},
$$

is the quantum-mechanical Hilbert space of the 4-component functions over $\mathrm{R}^{3} \subset \mathrm{M}(1,3)$ (depending parametrically on $x^{0}=t$ ), which are the square modulus integrable over the Lebesgue measure $\mathrm{d}^{3} x$ in the space $\mathrm{R}^{3} \subset \mathrm{M}(1,3)$. Just the space $\mathrm{R}^{3}$ is interpreted as the coordinate spectrum of the quantum-mechanical particles described by the field $\psi$.

In this concept, the Dirac equation in the Schrödinger form

$$
\mathrm{i} \partial_{0} \psi=H \psi \leftrightarrow\left(\partial_{0}-\widetilde{p}_{0}\right) \psi=0 ; \quad H \equiv \gamma^{0}\left(-\mathrm{i} \gamma^{k} \partial_{k}+m\right), \quad \widetilde{p}_{0}=-\mathrm{i} H,
$$

(which is completely equivalent to the equation (1)) is presented in its integral form as follows:

$$
\psi(t)=u\left(t_{0}, t\right) \psi\left(t_{0}\right) ; \quad u\left(t_{0}, t\right)=\exp \left[-\mathrm{i} H\left(t-t_{0}\right)\right] ; \quad \psi\left(t_{0}\right), \psi(t) \subset \mathrm{S}^{3,4 *} .
$$

In formula (15), the unitary operator $u\left(t_{0}, t\right)$ (with arbitrary-fixed parameters $t_{0}, t \in(-\infty, \infty) \subset$ $\mathrm{M}(1,3))$ is the operator of automorphism in the rigged Hilbert space (12) (below we set $t_{0}=0$ ).

Recall that the test function space $\mathrm{S}^{3,4}$ has a few wonderful features. It consists of the functions being infinitely smooth (infinitely differentiable with respect to $x^{\ell}$ ) rapidly decreasing at the infinity 
$|\vec{x}| \rightarrow \infty$ in arbitrary direction in $\mathrm{R}^{3}$ together with its derivatives of arbitrary orders. Further, it contains the space of the finite functions with the same properties. Moreover, the space $\mathrm{S}^{3,4}$ is kernel in the triple (12). The latter one means that this space is dense both in the $\mathrm{H}^{3,4}$ and $\mathrm{S}^{3,4 *}$ spaces. Therefore, hereinbelow we restrict our consideration to the suggestion $\psi \in \mathrm{S}^{3,4}$ in equation (14). Such restriction is both mathematically correct and technically appropriate (it does not require the use of the functional form of the elements $\psi \in \mathrm{S}^{3,4 *}$ ). It is also physically motivated. Indeed, an arbitrary measurement of a construction from $\psi^{\text {gen }} \subset S^{3,4 *}$ by an equipment of an arbitrary degree of accuracy can be successfully approximated (with directly fixing an arbitrarily precise degree of accuracy) by the corresponding constructions from the prelimit functions $\psi \in \mathrm{S}^{3,4} \subset \mathrm{H}^{3,4} \subset \mathrm{S}^{3,4 *}$.

For definiteness here we use the Pauli-Dirac (PD) representation of the Clifford-Dirac (CD) $\gamma$-matrices

$$
\begin{aligned}
& \gamma^{\mu}: \quad \gamma^{\mu} \gamma^{\nu}+\gamma^{\nu} \gamma^{\mu}=2 g^{\mu \nu}, \quad g=\left(g_{\nu}^{\mu}\right)=\operatorname{diag} g(+---), \\
& \gamma^{0}=\left|\begin{array}{ll}
1 & 0 \\
0 & -1
\end{array}\right|, \quad \gamma^{k}=\left|\begin{array}{ll}
0 & \sigma^{k} \\
-\sigma^{k} & 0
\end{array}\right| ; \quad \sigma^{1}=\left|\begin{array}{ll}
0 & 1 \\
1 & 0
\end{array}\right|, \\
& \sigma^{2}=\left|\begin{array}{ll}
0 & -i \\
i & 0
\end{array}\right|, \quad \sigma^{3}=\left|\begin{array}{ll}
1 & 0 \\
0 & -1
\end{array}\right|, \quad \ell=1,2,3 .
\end{aligned}
$$

In this representation, the $\mathrm{SU}(2)$-spin primary generators are given by the matrices $s_{\ell n} \equiv \frac{1}{4}\left[\gamma_{\ell}, \gamma_{n}\right]$. Two of them are quazidiagonal and the $s_{z}$-matrix is diagonal:

$$
\vec{s}=\left(s_{23}, s_{31}, s_{12}\right) \equiv\left(s^{\ell}\right)=-\frac{\mathrm{i}}{2}\left|\begin{array}{cc}
\vec{\sigma} & 0 \\
0 & \vec{\sigma}
\end{array}\right| \rightarrow s_{z} \equiv s^{3}=-\frac{\mathrm{i}}{2}\left|\begin{array}{cccc}
1 & 0 & 0 & 0 \\
0 & -1 & 0 & 0 \\
0 & 0 & 1 & 0 \\
0 & 0 & 0 & -1
\end{array}\right| .
$$

Therefore, the operator $s_{z}$ contains the projections of the spin $\vec{s}$ on the axis $\mathrm{z}$ of the quantummechanical spin- $\frac{1}{2}$ doublet of particles.

The primary $\mathcal{P}$-generators of the $\mathcal{P}^{\mathrm{F}}$-representation in the space $\mathrm{S}^{3,4}$ have the form of the following anti-Hermitian operators

$$
\begin{gathered}
\widetilde{p}_{0}=-\mathrm{i} H \equiv-\gamma_{0}\left(\gamma^{\ell} \partial_{l}+\mathrm{i} m\right), \quad \widetilde{p}_{k}=p_{k}=\partial_{k}, \\
\widetilde{j}_{k l}=j_{k l}=m_{k l}+s_{k l}, \quad \widetilde{j}_{o k}=t \partial_{k}-\frac{1}{2}\left\{x_{k}, \widetilde{p}_{0}\right\},
\end{gathered}
$$

where $\{\mathrm{A}, \mathrm{B}\} \equiv \mathrm{AB}+\mathrm{BA}$ and $t \in(-\infty, \infty)$ is the arbitrary-fixed parameter (the primary generators $\widetilde{q}=\left(\widetilde{p}_{\mu}, \widetilde{j}_{\mu \nu}\right)(19)$ coincide with the corresponding operators $-\mathrm{i} \widetilde{q}^{\text {stand }}$, where $\widetilde{q}^{\text {stand }}$ are given by the formulae (126)-(129) in [24]). Using the Heisenberg commutation relations in the form $\left[x^{\ell}, \partial_{j}\right]=\delta_{\ell j}$ and the $\mathrm{SU}(2)$-relations for $s^{\ell}(18)$, it is easy to show that the operators (19) satisfy the commutation relations of $\mathcal{P}$-algebra in the manifestly covariant form (2). Furthermore, generators (19) commute with the operator $\left(\partial_{0}-\widetilde{p}_{0}\right)$ of the equation $(14)$. Therefore, as a consequence of anti-Hermiticity of arbitrary operator from (19), the induced $\mathcal{P}^{\mathrm{F}}$-representation

$$
(a, \omega) \in \mathcal{P} \rightarrow \widetilde{\mathrm{U}}(a, \omega)=\exp \left(a^{\mu} \widetilde{p}_{\mu}+\frac{1}{2} \omega^{\mu \nu} \widetilde{j}_{\mu \nu}\right)
$$

in the space $S^{3,4}$ is unitary and is the group of invariance of equation (14). Hence, in the definition

$$
w^{\mu} \equiv \frac{1}{2} \varepsilon^{\mu \nu \rho \sigma} \widetilde{p}_{\rho} \widetilde{j}_{\nu \sigma} \rightarrow w^{0}=s^{\ell} \partial_{\ell} \equiv \vec{s}^{\text {stand }} \cdot \vec{p}^{\text {stand }}
$$

the main Casimir operators for the generators (19) are given by

$$
\begin{gathered}
\widetilde{p}^{\mu} \widetilde{p}_{\mu} \equiv \widetilde{p}_{0}^{2}-\partial_{\ell}^{2} \equiv m^{2} \mathrm{I}_{4}, \quad \mathrm{I}_{4} \equiv \operatorname{diag}(1,1,1,1) \\
\mathcal{W} \equiv w^{\mu} w_{\mu}=m^{2} \vec{s}^{2}=m^{2} \frac{1}{2}\left(\frac{1}{2}+1\right) \mathrm{I}_{4}
\end{gathered}
$$


According to the Bargman-Wigner classification, precisely this fact means that in equation (14) (for which the induced $\mathcal{P}^{\mathrm{F}}$-representation (20) is the group of invariance) the field $\psi$ is the Fermi field (the field of quantummechanical spin- $\frac{1}{2}$ doublet of particles with the mass $m$ ).

The relativistic invariance of the spinor field theory with respect to the representation (20) has some special features. It should be stressed once more that in the induced $\mathcal{P}^{\mathrm{F}}$-representation (20) the time $t=x^{0}$ plays a special role in comparison to the role of space variables $x^{\ell}$. Moreover, we use in the definition (13) such $\mathcal{P}$-non-covariant objects as the Lebesgue measures $d^{3} x$ (or $d^{3} k$ in the momentum representation of the rigged Hilbert space (12) for the field states $\psi \in \mathrm{S}^{3,4}$ ). Nevertheless, the theory of the spinor field $\psi$ based on the induced $\mathcal{P}^{\mathrm{F}}$-representation $(20)$ is obviously relativistic invariant. The proof is given in the text after formulae (19) and by the Bargman-Wigner analysis of the Casimir operators (22), (23).

Now we are in position to give some comparison of the local and induced $\mathcal{P}^{\mathrm{F}}$-representations (5) and (20). It is easy to see that in the set of solutions $\Psi=\{\psi\}$ of equations (14)=(1) the local $\mathcal{P}^{\mathrm{F}}$-representation (5) and the induced $\mathcal{P}^{\mathrm{F}}$-representation (20) coincide. Moreover, the main Casimir operators for the Lie $\mathcal{P}$-generators (4) have the form

$$
p^{\mathrm{loc} 2} \equiv p^{\mu} p_{\mu} \equiv \partial^{\mu} \partial_{\mu}, \quad \mathcal{W}^{\mathrm{loc}} \equiv m^{2} \vec{s}^{2}=\frac{3}{4} \partial^{\mu} \partial_{\mu}
$$

Therefore, the eigenvalues of the main Casimir operators for the $\mathcal{P}^{\mathrm{F}}$-representations (5) and (20) coincide. Note, by the way, that it is easy to show that formulae (24) have the same form for arbitrary Lie operators (4) (i.e., for arbitrary local $\mathcal{P}$-representations), for which the Lorentz spins $s_{\mu \nu}$ generate the $\mathcal{L}$-representations $(s, 0) \otimes(0, s)$.

Mark that the local generators (4) in $S^{4,4}$ are the functions of 14 independent operators $x^{\rho}, \partial_{\rho}, s_{\rho \sigma}$ (the Lorentz spin operators $s_{\rho \sigma}$ are the independent orts of the CD-algebra). The conception of Hermiticity or anti-Hermiticity in $\mathrm{S}^{4,4}$ is not inherent both in these 14 operators and $\mathcal{P}$-generators (4). Therefore, the concept of unitarity is not inherent in the local $\mathcal{P}^{\mathrm{F}}$-representation (5) as well. It means that the $\mathcal{P}^{\mathrm{F}}$-representation (5) itself (and, similarly, its generators (4)) does not contain the information as to "which quantum-mechanical particles are described by the filed $\psi$ from equation (1)?".

Contrary to these facts, the generators $(19)$ of induced $\mathcal{P}^{\mathrm{F}}$-representation in the space $\mathrm{S}^{3,4}$ are the anti-Hermitian functions of (particularly) other 11 independent operators $\vec{x}=\left(x^{\ell}\right), \partial_{\ell}$, $s_{k \ell}, \gamma^{0}, m$ or are the functions of the standard Hermitian in $\mathrm{S}^{3,4}$ operators: $\vec{x}=\left(x^{\ell}\right), \vec{p}^{\text {stand }}=$ $\left(-\mathrm{i} \partial_{\ell}\right), \vec{s}^{\text {stand }}=\left(\mathrm{i} s_{\ell n}\right), \gamma^{0}, m$. Hence, the induced $\mathcal{P}^{\mathrm{F}}$-representation $(20)$ is unitary in the quantummechanical rigged Hilbert space (12). However, the restriction of the local $\mathcal{P}^{\mathrm{F}}$-representation $(5)$ on the set $\Psi \subset \mathrm{S}^{3,4}$ of solutions of equation $(1)=(14)$ coincides with the induced $\mathcal{P}^{\mathrm{F}}$-representation $(20)$.

Nevertheless, both local and induced $\mathcal{P}^{\mathrm{F}}$-representations have some common physical shortcomings (namely this is the reason why we have started with the FW-representation of the spinor field theory, where $\mathcal{P}^{\mathrm{F}}$-representation is free from the above shortcomings). These shortcomings are related to the PD-representation of the spinor field $\psi$. In order to explain these assertions, we recall the general solution of equation $(14)=(1)$ in the rigged Hilbert space (12):

$$
\psi(x)=\frac{1}{(2 \pi)^{\frac{3}{2}}} \int \mathrm{d}^{3} k\left[a^{\mathrm{r}}(\vec{k}) v_{\mathrm{r}}^{-}(\vec{k}) \mathrm{e}^{-\mathrm{i} k x}+b^{* \mathrm{r}}(\vec{k}) v_{\mathrm{r}}^{+}(\vec{k}) \mathrm{e}^{\mathrm{i} k x}\right], \quad \mathrm{r}=1,2 .
$$

Here $k x \equiv \widetilde{\omega} t-\vec{k} \vec{x}, \quad \widetilde{\omega} \equiv \sqrt{\vec{k}^{2}+m^{2}}$ and the 4-component spinors are given by

$$
\begin{gathered}
v_{\mathrm{r}}^{-}(\vec{k})=N\left|\begin{array}{c}
(\widetilde{\omega}+m) \mathrm{d}_{\mathrm{r}} \\
(\vec{\sigma} \cdot \vec{k}) \mathrm{d}_{\mathrm{r}}
\end{array}\right|, \quad v_{\mathrm{r}}^{+}(\vec{k})=N\left|\begin{array}{c}
(\vec{\sigma} \cdot \vec{k}) \mathrm{d}_{\mathrm{r}} \\
(\widetilde{\omega}+m) \mathrm{d}_{\mathrm{r}}
\end{array}\right| ; \\
N \equiv \frac{1}{\sqrt{2 \widetilde{\omega}(\widetilde{\omega}+m)}}, \quad \mathrm{d}_{1}=\left|\begin{array}{c}
1 \\
0
\end{array}\right|, \quad \mathrm{d}_{2}=\left|\begin{array}{c}
0 \\
1
\end{array}\right| .
\end{gathered}
$$

We want to emphasize that, in general, the four independent amplitudes $a^{\mathrm{r}}(\vec{k}), b^{\mathrm{r}}(\vec{k})$ are the generalized functions from the rigged Hilbert space in the momentum representation. Still, if $\psi \in \mathrm{S}^{3,4} \subset$ 
$\mathrm{S}^{3,4 *}$, then $a^{\mathrm{r}}(\vec{k}), b^{\mathrm{r}}(\vec{k}) \in \widetilde{\mathrm{S}}^{3,4} \equiv \widetilde{\mathrm{S}}\left(\mathrm{R}_{\vec{k}}^{3}\right) \times \mathrm{C}^{4}$ (where $\mathrm{R}_{\vec{k}}^{3}$ is the spectrum of the quantummechanical operator $\vec{p}$ ) and the space $\widetilde{\mathrm{S}}^{3,4}$ is a kernel in the rigged Hilbert space $\widetilde{\mathrm{S}}^{3,4} \subset \mathrm{L}_{2}\left(\mathrm{R}_{\vec{k}}^{3}\right) \times \mathrm{C}^{4} \subset \widetilde{\mathrm{S}}^{3,4 *}$. Taking into account that any partner from the triplet of spaces (12) is invariant with respect to the 3-dimensional Fourier-transformation, one can omit the "tilde" symbol over these spaces. Therefore, exactly the fundamental solutions $\mathrm{e}^{\mp \mathrm{i} k x} v_{\mathrm{r}}^{\mp}(\vec{k})$ of the equations $(14)=(1)$ are the essentially generalized solutions (for them $\left.\left(a^{\mathrm{r}}(\vec{k}), b^{\mathrm{r}}(\vec{k})\right) \sim \delta\left(\vec{k}-\vec{k}^{\prime}\right) \in \mathrm{S}\left(\mathrm{R}_{\vec{k}}^{3}\right)^{*}\right)$.

It is well-known that the spinors $v_{\mathrm{r}}^{\mp}(\vec{k})(26)$ are called the spin states of the doublet. Nevertheless, they are not the eigenvectors of the operator $s_{z}=s^{3}$ from $\vec{s}(18)$. They are the eigenvectors of the operator $s_{z}^{\text {Dirac }} \equiv s^{3 \text { Dirac }} \subset \vec{s}^{\text {Dirac }}$ from nonlocal spin operator in the PD-representation for the spinor field $\psi$, which is presented by

$$
\vec{s}^{\text {Dirac }}=V^{-1} \vec{s} V=\vec{s}-\frac{\mathrm{i}[\vec{\gamma} \times \nabla]}{2 \widehat{\omega}}+\frac{\nabla \times[\vec{s} \times \nabla]}{\widehat{\omega}(\widehat{\omega}+m)}
$$

and which is obtained from the $\vec{s}$ (18) by the FW-transformation $V$ [18] (the prime operator $\vec{s}^{\text {Dirac }}(27)$ coincides with the corresponding spin $-\mathrm{i} \vec{s} \mathrm{FW}$, where $\vec{s} \mathrm{FW}$ is given in the table 1 in [18]). Indeed, it is easy to see that the following equations are valid:

$$
\begin{array}{rlrl}
s_{z} v_{1}^{\mp}(\vec{k}) & \neq \frac{1}{2} v_{1}^{\mp}(\vec{k}), & s_{z} v_{2}^{\mp}(\vec{k}) \neq-\frac{1}{2} v_{2}^{\mp}(\vec{k}) ; \\
s_{z}^{\text {Dirac }} v_{1}^{\mp}(\vec{k})=\frac{1}{2} v_{1}^{\mp}(\vec{k}), & s_{z}^{\operatorname{Dirac}} v_{2}^{\mp}(\vec{k})=-\frac{1}{2} v_{2}^{\mp}(\vec{k}),
\end{array}
$$

where $s_{z}=s^{3}(18), s_{z}^{\text {Dirac }}=s^{\text {Dirac } 3}(27)$ (the assertion (28) is our small addition to the consideration [18]). Moreover, operator $\vec{s}(18)$ does not commute with both the prime Dirac operator from equation (1) and the operator $\partial_{0}-\widetilde{p}_{0}$ (or $H$ ) from (14). Therefore, for the spinor field $\psi$ in the PD-representation the operator $\vec{s}(18)$ cannot be interpreted as the quantum-mechanical spin operator for the spin $\frac{1}{2}$-doublet of particles and does not generate the spin conservation law (even in the absence of interaction), which contradicts the experiment. Furthermore, as is known from [18], the operator $\vec{x}=\left(x^{\ell}\right) \in \mathrm{R}^{3} \subset \mathrm{M}(1,3)$ in the PD-representation for the field $\psi$ also cannot be interpreted as the quantum-mechanical operator of 3-coordinate for the spin $\frac{1}{2}$-doublet of particles. Nevertheless, operators $\vec{s}(18)$ and $\vec{x}$ are the important structure operators (which possess the physically meaningful quantum-mechanical spectra) for the construction of generators (4), (19) and corresponding local and induced $\mathcal{P}^{\mathrm{F}}$-representations.

As shown in [18] the above shortcomings follow from the non-diagonality of the Hamiltonian $H=\mathrm{i} \widetilde{p}_{0}$ in (14). Therefore, in the PD-representation of the field $\psi$, the particle and antiparticle states are mixed. The progressive way of moving forward was suggested in [18]. The FWrepresentation of the spinor field theory is free from the above shortcomings, e.g., the coordinate $\vec{x}=\left(x^{\ell}\right)$ of the FW-spinor $\phi$ and spin $\vec{s}(18)$ in this representation have direct physical meaning of corresponding quantum-mechanical observables of spin- $\frac{1}{2}$ doublet of particles. It is important that just the components of this spin $\vec{s}$ are the elements of the CD-algebra in the PD-representation. The above arguments impel us to start with the FW-representation of the spinor field (rather than with the PD-representation) and to consider the CD-algebra in the PD-representation just inside the FW-representation of the spinor field.

Our search for the hidden symmetries of the Dirac equation is based on the method of extension of the CD-algebra as the real one (based on the inclusion of the i and $C$ orts into it). This appears to be possible exactly owing to considering the $\mathcal{P}$-generators in terms of the primary operators.

\section{The Foldy-Wouthuysen representation and the extended real Clifford-Dirac algebra}

In order to derive the assertions mentioned in the introduction, we essentially use two constructive ideas (see Ansatz 1 and Ansatz 2 below). 
Ansatz 1. The above physical arguments (see section 2) impelled us to start not with the standard Dirac equation but with its FW representation [18]

$$
\begin{aligned}
&\left(\mathrm{i} \partial_{0}-\gamma_{0} \widehat{\omega}\right) \phi(x)=0, \phi=V \psi, \\
& V=\frac{-\mathrm{i} \vec{\gamma} \cdot \nabla+\widehat{\omega}+m}{\sqrt{2 \widehat{\omega}(\widehat{\omega}+m)}}, V^{-1}=V(\nabla \rightarrow-\nabla), \quad \widehat{\omega} \equiv \sqrt{-\Delta+m^{2}}, \quad \nabla \equiv\left(\partial_{\ell}\right) .
\end{aligned}
$$

In the space (12), the operator $V$ is unitary. The general solution of the FW-equation (29) (the Dirac equation in the FW-representation) in the quantum-mechanical rigged Hilbert space (12) is given by

$$
\phi(x)=V \psi(x)=\frac{1}{(2 \pi)^{\frac{3}{2}}} \int \mathrm{d}^{3} k\left[a^{\mathrm{r}}(\vec{k}) \mathrm{D}_{\mathrm{r}}^{-} \mathrm{e}^{-\mathrm{i} k x}+b^{* \mathrm{r}}(\vec{k}) \mathrm{D}_{\mathrm{r}}^{+} \mathrm{e}^{\mathrm{i} k x}\right], \quad \mathrm{r}=1,2,
$$

where the 4-dimensional Cartezian basis vectors in the space $\mathrm{C}^{4}$ have the form

$$
\mathrm{D}_{\mathrm{r}}^{-} \equiv V v_{\mathrm{r}}^{-}(\vec{k})=\left|\begin{array}{c}
\mathrm{d}_{\mathrm{r}} \\
0
\end{array}\right|, \quad \mathrm{D}_{\mathrm{r}}^{+} \equiv V v_{\mathrm{r}}^{+}(\vec{k})=\left|\begin{array}{c}
0 \\
\mathrm{~d}_{\mathrm{r}}
\end{array}\right|,
$$

and amplitudes $a^{\mathrm{r}}(\vec{k}), b^{\mathrm{r}}(\vec{k})$ as well as two-component vectors $\mathrm{d}_{\mathrm{r}}$ are the same as in $(25),(26)$. Contrary to the basis (26), the vectors (32) are the eigenvectors of the quantum-mechanical spin operator $s_{z} \in s^{3}$ from $\vec{s}$ (18). The fundamental solutions $\mathrm{e}^{\mp \mathrm{i} k x} \mathrm{D}_{\mathrm{r}}^{\mp}$ of equation (29) are the relativistic quantummechanical de Broglie waves (with the determined values of the spin $s_{z}$ from (18)) for the spin- $\frac{1}{2}$ doublet of particles.

Note that both the set of fundamental solutions $\left\{\Psi_{\vec{k} \mathrm{r}}^{\mp}\right\}$ of equation $(1)=(14)$ and $\left\{\Phi_{\vec{k} \mathrm{r}}^{\mp}\right\}$ of the equation (29),

$$
\Psi_{\vec{k} \mathrm{r}}^{\mp}(x) \equiv \frac{1}{(2 \pi)^{3 / 2}} \mathrm{e}^{\mp \mathrm{i} k x} v_{\mathrm{r}}^{\mp}(\vec{k}), \quad \Phi_{\vec{k} \mathrm{r}}^{\mp}(x) \equiv \frac{1}{(2 \pi)^{3 / 2}} \mathrm{e}^{\mp \mathrm{i} k x} \mathrm{D}_{\mathrm{r}}^{\mp},
$$

are the common eigenvectors of the complete set of mutually commute independent operators in $\mathrm{S}^{3,4}$, namely $\left(\vec{p}^{\text {stand }}=\left(-\mathrm{i} \partial_{\ell}\right), s_{z}^{\text {Dirac }}\right)$ for the set $\left\{\Psi_{\vec{k} \mathrm{r}}^{\mp}\right\}$ and $\left(\vec{p}^{\text {stand }}, s_{z}\right)$ for the set $\left\{\Phi_{\vec{k} \mathrm{r}}^{\mp}\right\}$ (they certainly satisfy one and the same orthonormality and completeness conditions). These facts present the evident proof of an adequate physical meaning of the spin operators $\vec{s}^{\text {Dirac }}(27)$ and $\vec{s}(18)$ : the first is the true spin for the spinor field $\psi$ in PD-representation and the second is the true spin for the spinor field $\phi$ in $\mathrm{FW}$-representation.

Moreover, now the $\mathcal{P}^{\mathrm{F}}$-generators of the unitary (induced) $\mathcal{P}^{\mathrm{F}}$-representation for the field $\phi$ (obtained from the operators (19) with the help of $V$-transformation (30)) are the functions of 11 directly experimentally observed operators $\left(\vec{x}=\left(x^{\ell}\right), \vec{p}^{\text {stand }}=\left(-\mathrm{i} \partial_{\ell}\right), \vec{s}^{\text {stand }}=\left(\mathrm{i} s_{\ell_{n}}\right), \gamma^{0}, m\right)$ or prime operators $\left(\vec{x}=\left(x^{\ell}\right), \nabla=\left(\partial_{\ell}\right), \vec{s}=\left(s^{\ell}\right)(18), \gamma^{0}, m\right)$ :

$$
\begin{aligned}
& \check{p}_{0}=-\mathrm{i} \gamma_{0} \widehat{\omega}, \quad \check{p}_{n}=\widetilde{p}_{n}=\partial_{n}, \\
& \check{j}_{l n}=\widetilde{j}_{l n}=x_{l} \partial_{n}-x_{n} \partial_{l}+s_{l n}, \quad \check{j}_{0 k}=x_{0} \partial_{k}+\mathrm{i} \gamma_{0}\left\{x_{k} \widehat{\omega}+\frac{\partial_{k}}{2 \widehat{\omega}}+\frac{(\vec{s} \times \vec{\partial})_{k}}{\widehat{\omega}+m}\right\}
\end{aligned}
$$

(the standard form of the primary generators (34) see e.g. in the formulae (D-64)-(D-67) in [24]). Now the unitary $\mathcal{P}^{\mathrm{F}}$-representation for the field $\phi$,

$$
(a, \omega) \in \mathcal{P} \rightarrow \check{\mathrm{U}}(a, \omega)=\exp \left(a^{\mu} \check{p}_{\mu}+\frac{1}{2} \omega^{\mu \nu} \check{j}_{\mu \nu}\right)
$$

(the $\mathcal{P}^{\mathrm{F}}$-group of invariance of the equation (29)) is free from the above mentioned shortcomings inherent in the local (3) and induced $(20) \mathcal{P}^{\mathrm{F}}$-representations. The Casimir operators for the generators (34) are the same as in (22), (23). The theory of the spinor field $\phi$ in the FW-representation, 
which is recalled here, can be evidently generalized for the fields of any spin $\vec{s}$ (where $\left(s^{\ell}\right) \equiv \vec{s}$ are the arbitrary generators of an $\mathrm{SU}(2)$-algebra representation).

Not a matter of special separate role of the time variable $t=x^{0}$, the theory of the spinor field $\phi$ in the FW-representation is the relativistic invariant (namely, $\mathcal{P}$-invariant) in the sense of two necessary aspects. (i) The dynamical aspect: the unitary $\mathcal{P}^{\mathrm{F}}$-representation (35) is the group of invariance of the equation (29) (the main conservation laws for the spinor field $\phi$ in the FWrepresentation are the consequences of this assertion). (ii) The kinematical aspect: if the solution (31) with arbitrary-fixed amplitudes $a^{\mathrm{r}}(\vec{k}), b^{\mathrm{r}}(\vec{k})$ is a given state of the spinor field $\phi$ in the arbitrary-fixed inertial frame of references (IFR) $\Sigma$, then for the observer in the $(a, \omega)$-transformed IFR $\Sigma^{\prime}$ the solution $\phi^{\prime}(x)=\check{\mathrm{U}}(a, \omega) \phi(x), x \in \mathrm{M}(1,3)$, is the same state of the spinor field.

Some other details of physical arguments for our start with the FW-representation are as follows. Just the components of the field $\phi$ of the FW equation (29) coincide with the quantummechanical wave functions of the particle doublet. Furthermore, it is the FW-representation, where the operators of directly experimentally observed quantities of this doublet are the corresponding direct sums of the quantum-mechanical observables of single particles, which form the doublet. Therefore, it is in the canonical (i.e. FW) representation where these operators have the status of true observables of the particle doublet. In particular, the 16-dimensional Clifford-Dirac (CD) algebra (see formulae (36) below) generated by the $\gamma^{\mu}$-matrices (17) contains the generators of the $\mathrm{SU}(2)$ group, which commute with the Hamiltonian $\gamma^{0} \widehat{\omega}$ of the FW equation (29). The latter assertion means that the spin $\vec{s}$ (18) of the free particle doublet is conserved. Therefore, the operator $\vec{s}$ (18) given in the set $\{\phi\}$ of solutions (31) of equation (29) has the status of true spin. This is the reason to consider the prime CD algebra in the PD representation (17) as an algebra in the set $\{\phi\} \subset(12)$. In order to avoid misunderstandings, note that in our consideration the $\gamma^{\mu}$-matrices in the FW representation have the form (17), rather than $V \gamma^{\mu} V^{-1}$. Of course, $V^{-1}\left(\mathrm{i} \partial_{0}-\gamma_{0} \widehat{\omega}\right) V=\mathrm{i} \partial_{0}-H$ with the $\gamma^{\mu}$-matrices (17).

Finally, one has a simple technical reason to prefer the FW-representation. In the FW-representation the operator of equation (29) contains only one diagonal $\gamma^{0}$-matrix instead of four $\gamma^{\mu}$ matrices in equation $(1)=(14)$. Hence, equation (29) and the FW-representation are much more convenient in searching for the matrix symmetries (in comparison with the prime equation $(1)=(14)$ and the PD-representation for the spinor field). Note that this method and the advantages of the FW-representation have been well-known since the appearance of publications [18, 24] (see e.g. the table 1 in [18], where the physically important symmetry operator $\vec{s}^{\text {Dirac }}(27)$ was found, or the generators (126)-(129) in 24]). We recall them only for our purposes.

Ansatz 2. Here (see the formula (38) below) we introduce a 64-dimensional extended real Clifford-Dirac algebra (ERCD algebra) as the algebra of $4 \times 4$ pure matrix operators in the set $\{\phi\} \subset(12)$. We essentially apply it here as constructive mathematics for our purposes. For the physical purposes, where the parameters of the relativistic groups are real, it is sufficient to consider the 16-dimensional standard CD algebra in the complex space (12) as real algebra. It enables us, along with the application of the imaginary unit "i" and the operator $\hat{C}$ of complex conjugation in the set $\{\phi\} \subset(12)$, to extend this algebra to the ERCD algebra in the space (12). It is on the basis of the ERCD algebra that we are able to find the maximal pure matrix algebra (without the space-time derivatives $\partial_{\mu}$ ) of invariance of the FW equation and the corresponding algebra of invariance of the Dirac equation with an arbitrary mass in the standard PD representation. The ERCD algebra is the complete set of operators, the part of which generates the Pauli-GurseyIbragimov (PGI) algebra. Let us recall that the 8-dimensional (for $m=0$ ) and 4-dimensional (for a nonzero mass) PGI algebras of invariance of the Dirac equation are considered in detail, for instance, in [13, 17] (these algebras were put into consideration in the original papers by W. Pauli, F. Gursey and N. Ibragimov, see, e.g. [25, 26]).

Finally, it is useful to choose 16 independent (ind) generators - orts of standard CD algebra as

$$
\begin{aligned}
& \{\operatorname{indCD}\} \equiv\left\{\mathrm{I}, \quad \alpha_{\hat{\mu} \hat{\nu}}=2 s_{\hat{\mu} \hat{\nu}}: s_{\breve{\mu} \check{\nu}} \equiv \frac{1}{4}\left[\gamma_{\check{\mu}}, \gamma_{\check{\nu}}\right], \quad s_{\breve{\mu} 5}=-s_{5 \check{\mu}} \equiv \frac{1}{2} \gamma_{\check{\mu}} ;\right. \\
& \left.\gamma_{4} \equiv \gamma_{0} \gamma_{1} \gamma_{2} \gamma_{3}, \quad \hat{\mu}, \hat{\nu}=\overline{0,5}, \quad \check{\mu}, \check{\nu}=\overline{0,4}\right\},
\end{aligned}
$$


where $\gamma_{4} \equiv \gamma_{0} \gamma_{1} \gamma_{2} \gamma_{3}=\mathrm{i} \gamma_{5}^{\text {stand }}$ and the matrices $s_{\hat{\mu} \hat{\nu}}$ are the prime generators of the $\operatorname{SO}(1,5) \supset \operatorname{SO}(1,3)=$ $\mathrm{L}_{+}^{\uparrow}$ group (associated with the real parameters - the angles $\omega^{\hat{\mu} \hat{\nu}}$ of rotations in $\hat{\mu} \hat{\nu}$ planes of the space $\mathrm{M}(1,5) \supset \mathrm{M}(1,3))$, these operators satisfy the following commutation relations

$$
\begin{aligned}
{\left[s_{\hat{\mu} \hat{\nu}}, s_{\hat{\rho} \hat{\sigma}}\right] } & =-g_{\hat{\mu} \hat{\rho}} s_{\hat{\nu} \hat{\sigma}}-g_{\hat{\rho} \hat{\nu}} s_{\hat{\sigma} \hat{\mu}}-g_{\hat{\nu} \hat{\sigma}} s_{\hat{\mu} \hat{\rho}}-g_{\hat{\sigma} \hat{\mu}} s_{\hat{\rho} \hat{\nu}} ; \quad \hat{\mu}, \hat{\nu}=\overline{0,5} \\
\left(g_{\hat{\nu}}^{\hat{\mu}}\right) & =\operatorname{diag}(+1,-1,-1,-1,-1,-1) .
\end{aligned}
$$

By complementing the orts (36) of the real CD algebra with the operators i, $\hat{C}$ and with all possible products of orts (36) and operators i and $\hat{C}$, we define the ERCD algebra as the linear manifold spanned on the orts

$$
\{\mathrm{ERCD}\}=\{\text { indCD }, \mathrm{i} \cdot \text { indCD }, \hat{C} \cdot \operatorname{indCD}, \mathrm{i} \hat{C} \cdot \operatorname{indCD}\} .
$$

Thus, the ERCD algebra generators are the compositions of the standard CD algebra generators (36) and the generators of the PGI algebra 25, 26], i.e. it is the maximal set of independent matrices, which can be constructed from the elements i, $\hat{C}$, and (36).

All the physically meaningful symmetries of the FW and Dirac equations put into consideration below are constructed using the elements of the ERCD algebra.

Note that all elements $\mathrm{S}^{3,4}, \mathrm{H}^{3,4}, \mathrm{~S}^{3,4 *}$ of the rigged Hilbert space (12) are the spaces with involution. It means that together with the element $f$ the rigged Hilbert space also contains the complex conjugated element $f^{*}=\hat{C} f$. Namely this circumstance (together with the comprehension of the main physical symmetry groups $\mathcal{P} \supset \mathcal{L}$, its subgroups and corresponding algebras as real spaces, i.e., the spaces over the field of real numbers, to which the parameters of the symmetry groups belong) makes it possible to consider and search for the hidden symmetries of the Dirac equation in the extension of the matrix algebras as the real ones due to adding the operators i and $\hat{C}$ to the orts of these algebras. In this context, the title ERCD algebra illustrates the fact that for both algebras (for CD algebra (36) as well as for ERCD algebra (38)) the part of the orts pairs commutes or anticommutes between each other and, furthermore, the square of every ort is equal to +1 or to -1 .

\section{Maximal pure matrix algebra of invariance of the Foldy-Wouthuysen equation}

Consider the 32-dimensional subalgebra $\mathrm{A}_{32}=\mathrm{SO}(6) \oplus \hat{\varepsilon} \cdot \mathrm{SO}(6) \oplus \hat{\varepsilon}$ of the ERCD algebra. It is easy to see that the part of ERCD algebra generators, namely the operators

$$
s_{A B}=\frac{1}{4}\left[\gamma_{A}, \gamma_{B}\right]=-s_{B A}, \quad A, B=\overline{1,6}, \quad \gamma_{5} \equiv \gamma_{1} \gamma_{3} \hat{C}, \quad \gamma_{6} \equiv i \gamma_{1} \gamma_{3} \hat{C},
$$

(here $A, B=\overline{1,6}$ includes $\check{a} \breve{b}=\overline{1,4}$ from (36)) and $\hat{\varepsilon}=\mathrm{i} \gamma_{0}$, satisfy the commutation relations

$$
\begin{aligned}
{\left[s_{A B}, s_{C D}\right] } & =\delta_{A C} s_{B D}+\delta_{C B} s_{D A}+\delta_{B D} s_{A C}+\delta_{D A} s_{C B}, \\
{\left[s_{A B}, \hat{\varepsilon}\right] } & =0 ; \quad A, B, C, D=\overline{1,6} .
\end{aligned}
$$

Therefore, operators (39) generate a representation of the $\mathrm{SO}(6) \supset \mathrm{SO}(3)$ group of space rotations in the space $\mathrm{R}^{6} \subset \mathrm{M}(1,6) \supset \mathrm{M}(1,3)$. On this basis, together with the additional $\mathrm{SO}(6)$ Casimir operator $\hat{\varepsilon} \equiv \mathrm{i} \gamma_{0}=-\gamma_{1} \gamma_{2} \gamma_{3} \gamma_{4} \gamma_{5} \gamma_{6}$, we define the $\mathrm{SO}(6) \oplus \hat{\varepsilon}$ algebra and, finally, the $\mathrm{A}_{32}=\mathrm{SO}(6) \oplus$ $\hat{\varepsilon} \cdot \mathrm{SO}(6) \oplus \hat{\varepsilon}$ algebra. The commutation relations for the $\hat{\varepsilon} \cdot \mathrm{SO}(6)$ generators $\tilde{s}_{A B}=\hat{\varepsilon} s_{A B}$ differ from (40) by the common factor $\hat{\varepsilon}=\mathrm{i} \gamma_{0}$. The 32-dimensional subalgebra $\boldsymbol{A}_{32}=\boldsymbol{S O}(\boldsymbol{6}) \oplus \hat{\varepsilon} \cdot \boldsymbol{S O}(\boldsymbol{6}) \oplus \hat{\varepsilon}$ of the ERCD algebra in the rigged Hilbert space (12) is the maximal pure matrix algebra (without the space-time derivatives $\partial_{\mu}$ ) of invariance of the $\boldsymbol{F} W$-equation (29).

The proof of this assertion is carried out by straightforward calculations of (i) the corresponding commutation relations (40) for elements of this algebra, (ii) the commutators between 
the elements of $\mathrm{A}_{32}$ and the operator $\left(\mathrm{i} \partial_{0}-\gamma_{0} \hat{\omega}\right)$ of the FW equation (29). The maximality of $\mathrm{A}_{32}$ as the algebra of invariance of the equation (29) is the consequence of the maximality of the $\operatorname{dim}(\mathrm{ERCD})=64$ (in the class of pure matrix operators).

Note that antihermitian matrices $\left\{\gamma_{A}: \gamma_{1}, \gamma_{2}, \gamma_{3}, \gamma_{4} \equiv \gamma_{0} \gamma_{1} \gamma_{2} \gamma_{3}, \gamma_{5} \equiv \gamma_{1} \gamma_{3} \hat{C}, \gamma_{6} \equiv \mathrm{i} \gamma_{1} \gamma_{3} \hat{C}\right.$, $\left.\gamma_{7} \equiv \mathrm{i} \gamma_{0}\right\}$ satisfy the commutation relations $\gamma_{A} \gamma_{B}+\gamma_{B} \gamma_{A}=-2 \delta_{A B}, A, B=\overline{1,7}$.

The explicit form of the elements of corresponding algebra $\mathrm{A}_{32}$ of invariance of the Dirac equation in the PD-representation is found from the elements (39) and $\hat{\varepsilon}$ with the help of the FW-transformation (30): $V^{-1}\left(\mathrm{~A}_{32}, \hat{\varepsilon}\right) V$. In PD-representation this algebra of invariance (of the prime Dirac equation (1)) is given by the nonlocal operators.

\section{Spin 1 Lorentz-symmetries of the Foldy-Wouthuysen and Dirac equations}

The FW-equation (29) is invariant with respect to the two different spin 1 representations of the Lorentz group $\mathcal{L}$ (below $s_{\mu \nu}^{\mathrm{V}}$ are the generators of the irreducible vector $(1 / 2,1 / 2)$ representation and $s_{\mu \nu}^{\mathrm{TS}}$ are the generators of the reducible tensor-scalar $(1,0) \oplus(0,0)$ representation of the $\operatorname{SO}(1,3)=\mathrm{L}_{+}^{\uparrow}$ algebra). The explicit forms of the corresponding pure matrix operators are given by

$$
s_{\mu \nu}^{\mathrm{TS}}=\left\{s_{0 k}^{\mathrm{TS}}=s_{0 k}^{\mathrm{I}}+s_{0 k}^{\mathrm{II}}, \quad s_{m n}^{\mathrm{TS}}=s_{m n}^{\mathrm{I}}+s_{m n}^{\mathrm{II}}\right\}, \quad s_{\mu \nu}^{\mathrm{V}}=\left\{s_{0 k}^{\mathrm{V}}=-s_{0 k}^{\mathrm{I}}+s_{0 k}^{\mathrm{II}}, \quad s_{m n}^{\mathrm{V}}=s_{m n}^{\mathrm{TS}}\right\},
$$

where $\mathrm{s}_{\mu \nu}^{\mathrm{I}, \mathrm{II}}$ are the following elements of $\mathrm{A}_{32}$ algebra:

$$
\begin{aligned}
s_{\mu \nu}^{\mathrm{I}}= & \left\{s_{0 k}^{\mathrm{I}}=\frac{\mathrm{i}}{2} \gamma_{4} \gamma_{k}, \quad s_{m k}^{\mathrm{I}}=\frac{1}{4}\left[\gamma_{m}, \gamma_{k}\right]\right\}, \quad \gamma_{4} \equiv \gamma_{0} \gamma_{1} \gamma_{2} \gamma_{3}, \quad(k, m=\overline{1,3}), \\
s_{\mu \nu}^{\mathrm{II}}= & \left\{s_{01}^{\mathrm{II}}=\frac{\mathrm{i}}{2} \gamma_{2} \hat{C}, \quad s_{02}^{\mathrm{II}}=-\frac{1}{2} \gamma_{2} \hat{C}, \quad s_{03}^{\mathrm{II}}=\frac{1}{2} \gamma_{0},\right. \\
& \left.s_{12}^{\mathrm{II}}=\frac{\mathrm{i}}{2}, \quad s_{31}^{\mathrm{II}}=\frac{\mathrm{i}}{2} \gamma_{2} \gamma_{0} \hat{C}, \quad s_{23}^{\mathrm{II}}=\frac{1}{2} \gamma_{2} \gamma_{0} \hat{C}\right\} .
\end{aligned}
$$

The sets $\mathrm{s}_{\mu \nu}^{\mathrm{I}}(42)$ and $\mathrm{s}_{\mu \nu}^{\mathrm{II}}$ (43) determine in $\mathrm{A}_{32}$ the generators of two different versions of $(1 / 2,0) \oplus$ $(0,1 / 2)$ representation of the $\mathcal{L}$-algebra. The validity of these assertions are evident after the transition $s_{\mu \nu}^{\text {Bose }}=W s_{\mu \nu}^{\mathrm{V}, \mathrm{TS}} W^{-1}$ to the Bose-representation of the $\gamma$-matrices $\left(\gamma^{\mu \text { Bose }}=W \gamma^{\mu} W^{-1}\right)$, where the operator $W$ is given by

$$
W=\frac{1}{\sqrt{2}}\left|\begin{array}{cccc}
0 & -1 & 0 & \hat{C} \\
0 & i & 0 & i \hat{C} \\
-1 & 0 & \hat{C} & 0 \\
-1 & 0 & -\hat{C} & 0
\end{array}\right|, \quad W^{-1}=\frac{1}{\sqrt{2}}\left|\begin{array}{cccc}
0 & 0 & -1 & -1 \\
-1 & -i & 0 & 0 \\
0 & 0 & \hat{C} & -\hat{C} \\
\hat{C} & i \hat{C} & 0 & 0
\end{array}\right|, \quad W W^{-1}=W^{-1} W=1
$$

In such Bose-representation, the $\gamma^{\mu \text { Bose }}=W \gamma^{\mu} W^{-1}$ matrices contain not only the operator i, but also the operator $\hat{C}$ of complex conjugation:

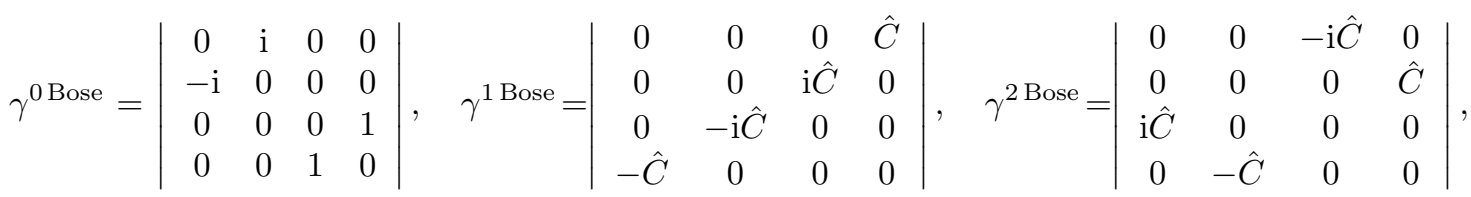

$$
\begin{aligned}
& \gamma^{3 \text { Bose }}=\left|\begin{array}{cccc}
0 & \mathrm{i} \hat{C} & 0 & 0 \\
-\mathrm{i} \hat{C} & 0 & 0 & 0 \\
0 & 0 & 0 & \hat{C} \\
0 & 0 & -\hat{C} & 0
\end{array}\right|, \quad \mathrm{i}^{\text {Bose }}=\left|\begin{array}{cccc}
0 & -1 & 0 & 0 \\
1 & 0 & 0 & 0 \\
0 & 0 & 0 & \mathrm{i} \\
0 & 0 & \mathrm{i} & 0
\end{array}\right|, \quad \hat{C}^{\text {Bose }}=\left|\begin{array}{cccc}
\hat{C} & 0 & 0 & 0 \\
0 & -\hat{C} & 0 & 0 \\
0 & 0 & \hat{C} & 0 \\
0 & 0 & 0 & \hat{C}
\end{array}\right| \text {, }
\end{aligned}
$$

(of course, they satisfy the CD-relations (16)). Calculation of operator constructions (41) also needs the explicit forms of operators $\mathrm{i}^{\text {Bose }}, \hat{C}^{\text {Bose }}$. Therefore, we present them in (45) too. Now, 
together with $\gamma^{\mu \text { Bose }}$-matrices, they are not so simple as $\mathrm{iI}_{4}, \hat{C} \mathrm{I}_{4}$ in standard PD-representation ( $\mathrm{I}_{4}$ is $4 \times 4$ unit matrix). Nevertheless, we note, the Lorentz spin matrices $s_{\mu \nu}^{\mathrm{Bose}}=W s_{\mu \nu}^{\mathrm{V}, \mathrm{TS}^{-1}} W^{-1}$ in the Bose-representation do not contain the operator $\hat{C}$ and take the explicit forms well known for the matrix representations $(1,0) \otimes(0,0)$ and $(1 / 2,1 / 2)$ of the group $\mathcal{L} \sim \operatorname{SO}(1,3)$ :

$$
\begin{aligned}
& s_{12}^{\mathrm{V}, \mathrm{TS} \text { Bose }}=\left|\begin{array}{cccc}
0 & -1 & 0 & 0 \\
1 & 0 & 0 & 0 \\
0 & 0 & 0 & 0 \\
0 & 0 & 0 & 0
\end{array}\right|, \quad s_{31}^{\mathrm{V}, \mathrm{TS} \text { Bose }}=\left|\begin{array}{cccc}
0 & 0 & 1 & 0 \\
0 & 0 & 0 & 0 \\
-1 & 0 & 0 & 0 \\
0 & 0 & 0 & 0
\end{array}\right|, \quad s_{23}^{\mathrm{V}, \mathrm{TS} \text { Bose }}=\left|\begin{array}{cccc}
0 & 0 & 0 & 0 \\
0 & 0 & -1 & 0 \\
0 & 1 & 0 & 0 \\
0 & 0 & 0 & 0
\end{array}\right| \text {, } \\
& s_{01}^{\mathrm{TS} B o s e}=\left|\begin{array}{cccc}
0 & 0 & 0 & 0 \\
0 & 0 & -i & 0 \\
0 & i & 0 & 0 \\
0 & 0 & 0 & 0
\end{array}\right|, \quad s_{02}^{\mathrm{TS} B o s e}=\left|\begin{array}{cccc}
0 & 0 & i & 0 \\
0 & 0 & 0 & 0 \\
-i & 0 & 0 & 0 \\
0 & 0 & 0 & 0
\end{array}\right|, \quad s_{03}^{\mathrm{TS} B o s e}=\left|\begin{array}{cccc}
0 & -i & 0 & 0 \\
i & 0 & 0 & 0 \\
0 & 0 & 0 & 0 \\
0 & 0 & 0 & 0
\end{array}\right|, \\
& s_{01}^{\mathrm{V} \text { Bose }}=\left|\begin{array}{cccc}
0 & 0 & 0 & -1 \\
0 & 0 & 0 & 0 \\
0 & 0 & 0 & 0 \\
-1 & 0 & 0 & 0
\end{array}\right|, \quad s_{02}^{\mathrm{V} \text { Bose }}=\left|\begin{array}{cccc}
0 & 0 & 0 & 0 \\
0 & 0 & 0 & -1 \\
0 & 0 & 0 & 0 \\
0 & -1 & 0 & 0
\end{array}\right|, \quad s_{03}^{\mathrm{V} \text { Bose }}=\left|\begin{array}{cccc}
0 & 0 & 0 & 0 \\
0 & 0 & 0 & 0 \\
0 & 0 & 0 & -1 \\
0 & 0 & -1 & 0
\end{array}\right| .
\end{aligned}
$$

We refer to the transition $\phi \rightarrow W \phi$ with $W$ (44), which links the present fermionic and bosonic multipletes, as a new natural form of the supersymmetry transformation. The corresponding symmetries of the Dirac equation (1) are obtained using FW-transformation (30).

\section{Spin 1 Poincaré-symmetries of the Foldy-Wouthuysen equation}

The $\boldsymbol{F W}$ equation (29) is invariant not only with respect to the well-known standard spin $1 / 2 \mathcal{P}^{\mathrm{F}}$-representation (35), but also with respect to the canonical-type spin 1 representation (Bose representation) of the Poincaré group $\mathcal{P}$, i.e. with respect to the unitary (in the set $\{\phi\}$ of solutions of equation (29)) $\mathcal{P}^{\mathrm{B}}$-representation, which is determined by the primary generators

$$
\begin{aligned}
\widehat{p}_{0} & =\check{p}_{0}=-\mathrm{i} \gamma_{0} \widehat{\omega}, \quad \widehat{p}_{n}=\check{p}_{n}=\partial_{n}, \quad \widehat{j}_{l n}=x_{l} \partial_{n}-x_{n} \partial_{l}+s_{l n}^{\mathrm{I}}+s_{l n}^{\mathrm{II}}, \\
\widehat{j}_{0 k} & =x_{0} \partial_{k}+\mathrm{i} \gamma_{0}\left\{x_{k} \widehat{\omega}+\frac{\partial_{k}}{2 \widehat{\omega}}+\frac{\left[\left(\vec{s}^{\mathrm{I}}+\vec{s}^{\mathrm{II}}\right) \times \vec{\partial}\right]_{k}}{\widehat{\omega}+m}\right\},
\end{aligned}
$$

where $\widehat{\omega}$ is given in $(30), s_{l n}^{\mathrm{I}}$ and $s_{l n}^{\mathrm{II}}$ are given in $(42),(43)$, respectively, and $\vec{s}^{\mathrm{I}, \mathrm{II}}=\left(s_{23}, s_{31}, s_{12}\right)^{\mathrm{I}, \mathrm{II}}$.

The proof is performed by straightforward calculations of (i) the corresponding $\mathcal{P}$-commutators (2) between the generators (47), (ii) the commutators between generators (47) and operator ( $\mathrm{i} \partial_{0}-$ $\left.\gamma_{0} \hat{\omega}\right)$, (iii) the Casimir operators of the Poincaré group for the generators (47). According to the Bargman-Wigner classification of the $\mathcal{P}$-covariant fields, just these facts (especially (iii)) visualize the hidden Bose essence of the $\mathcal{P}^{\mathrm{B}}$-representation, generated by the operators (47). For the $\mathcal{P}^{\mathrm{B}}$ representation the explicit form of main Casimir operators as follows:

$$
\widehat{p}^{\mu} \widehat{p}_{\mu}=m^{2}, \quad \mathcal{W}^{\mathrm{B}}=w^{\mu} w_{\mu}=m^{2}\left(\vec{s}^{\mathrm{TS}}\right)^{2},
$$

(compare with (22), (23)), where $w^{\mu} \equiv \frac{1}{2} \varepsilon^{\mu \nu \rho \sigma} \widehat{p}_{\rho} \widehat{j}_{\nu \sigma}$ and, after diagonalization carried out with the help of operator $W(44)$,

$$
\left(\vec{s}^{\mathrm{TS}}\right)^{2}=\left(\vec{s}^{\mathrm{TS} \text { Bose }}\right)^{2}=-1(1+1)\left|\begin{array}{cc}
\mathrm{I}_{3} & 0 \\
0 & 0
\end{array}\right|, \quad \mathrm{I}_{3} \equiv\left|\begin{array}{ccc}
1 & 0 & 0 \\
0 & 1 & 0 \\
0 & 0 & 1
\end{array}\right| .
$$




\section{Spin 1 symmetries of the Dirac equation with nonzero mass}

It is easy to see that the prime Dirac equation (1) has all the above mentioned spin 1 symmetries of the $\boldsymbol{F} \boldsymbol{W}$ equation. The corresponding explicit forms of the generators $q^{\mathrm{PD}}$ in the manifold $\{\psi\}$ are obtained from the corresponding formulae (39), (41)-(43), (47) for the FW generators $q^{\mathrm{FW}}$ with the help of the FW operator $V(30): q^{\mathrm{PD}}=V^{-1} q^{\mathrm{FW}} V$. As a meaningful example, we present here the explicit form for the spin 1 generators of $\mathcal{P}^{\mathrm{B}}$-symmetries of the Dirac equation

$$
\begin{aligned}
& \widehat{p}_{0}^{\mathrm{PD}}=\widetilde{p}_{0}=-\mathrm{i} H, \quad \widehat{p}_{k}^{\mathrm{PD}}=\partial_{k}, \\
& \widehat{j}_{k l}^{\mathrm{PD}}=x_{k} \partial_{l}-x_{l} \partial_{k}+s_{k l}+\hat{s}_{k l}, \quad \widehat{j}_{0 k}^{\mathrm{PD}}=x_{0} \partial_{k}-x_{k} \widetilde{p}_{0}+s_{0 k}+\frac{\varepsilon_{k \ell n} \hat{s}_{0 \ell} \partial_{n}}{\widehat{\omega}+m},
\end{aligned}
$$

where $\varepsilon_{k l n}$ is the Levi-Chivitta tensor, and the operators $s_{\mu \nu}, \hat{s}_{\mu \nu}=V^{-1} s_{\mu \nu}^{\mathrm{II}} V$ have the form

$$
\begin{aligned}
& s_{\mu \nu}= \frac{1}{4}\left[\gamma_{\mu}, \gamma_{\nu}\right], \quad \mu, \nu=\overline{0,3}, \\
& \hat{s}_{\mu \nu}=\left\{\hat{s}_{01}=\frac{1}{2} \mathrm{i} \gamma_{2} \hat{C}, \quad \hat{s}_{02}=-\frac{1}{2} \gamma_{2} \hat{C}, \quad \hat{s}_{03}=\gamma^{0} \frac{\vec{\gamma} \cdot \vec{p}^{\text {stand }}+m}{2 \widehat{\omega}}=\frac{H}{2 \widehat{\omega}},\right. \\
&\left.\hat{s}_{12}=\frac{\mathrm{i}}{2}, \quad \hat{s}_{31}=-\frac{\mathrm{i} H}{2 \widehat{\omega}} \gamma_{2} \hat{C}, \quad \hat{s}_{23}=-\frac{H}{2 \widehat{\omega}} \gamma_{2} \hat{C}\right\}
\end{aligned}
$$

(the part of the Lorentz spin operators from (51) is not a pure matrix because they depend on the pseudodifferential operator $\widehat{\omega} \equiv \sqrt{-\Delta+m^{2}}$ well-defined in the space $\mathrm{S}^{3,4}$ ). Of course, the Casimir operators for the $\mathcal{P}^{\mathrm{B}}$-generators (50) have the same final form (48), (49) as for the generators (47). The Dirac equation in the Bose-representation of the $\gamma$-matrices $\left(\gamma^{\mu \text { Bose }}=W \gamma^{\mu} W^{-1}\right)$ is the Maxwell-type equation for a massive tensor-scalar field.

Note that the generators $(47)$ without the additional terms $s_{l n}^{\mathrm{II}}(43)$ and $\vec{s}^{\mathrm{II}}=\left(s_{23}, s_{31}, s_{12}\right)^{\mathrm{II}}$ directly coincide with the well-known generators (34) of standard Fermi (spin 1/2) $\mathcal{P}^{\mathrm{F}}$-symmetries of the FW-equation (similar situation occurs for the generators (50) taken without the terms including the operators $\hat{s}_{\mu \nu}$ from (51) - they coincide with the operators (19) of the induced $\mathcal{P}^{\mathrm{F}}$-representation (20)). These well-known forms determine the Fermi-case, while the operators suggested here are related to the Bose interpretation of equations (1), (29), which is found here also to be possible. The only difference of our Fermi-case from the spin $1 / 2$ generators in [18] is that we use the prime form of generators related to the real parameters of the Poincaré group.

\section{The case of zero mass, brief remix}

Having analysed the arbitrary mass case presented here, the specific character of the zero mass case [12 17] becomes evident. For $m=0$ the analogues of the additional operators $\hat{s}_{\mu \nu}(51)$ are the local pure matrix Lie operators (see, e.g. formulae (12) in [13]). Therefore, for the $m=0$ case, the corresponding $\mathcal{P}^{\mathrm{B}}$-generators are much simpler than those in (50) and contain no nonlocal terms. Moreover, for the $m=0$ case, there is no need to turn to the FW-representation and ERCD algebra. In 12 17 all Bose symmetries of the massless Dirac equation were found in the standard PD-representation of the spinor field $\psi$ on the basis of the ordinary CD algebra and well-known [25, 26] PGI operators. Thus, the search for the additional bosonic symmetries of the massless Dirac equation is technically much easier. Therefore, our results [12 17] contain a lot of additional meaningful information in comparison with the results presented here for the nonzero mass. In 12 17], the full consideration of the Fermi-Bose duality of the massless Dirac equation is presented. Furthermore, the Fermi-Bose duality of the Maxwell equations with the gradient-type sources, which are the Bose partner of the massless Dirac equation, is investigated in detail. In our further publications we will be in position to extend and generalize on the same level all our results presented briefly here and to reproduce all the results of [12 17] for the case of nonzero mass. For 
example, the unitary relationship between the fermionic amplitudes $a^{\mathrm{r}}(\vec{k}), b^{\mathrm{r}}(\vec{k})$ of the solution (25) and the bosonic amplitudes of the general solution of the Maxwell-type equation for a massive tensor-scalar field will be presented (the analogue of the unitary relationship (31) in [15], or (40) in [16], from the case $m=0)$. Hence, the subsequent steps of the analysis of the Fermi-Bose duality of the Dirac equation with arbitrary mass will be presented.

\section{Brief conclusions}

The following four principal results have been proved and presented.

1. In the FW-representation for the Dirac equation the new mathematical object - the 64dimensional Extended real Clifford-Dirac (ERCD) algebra - is put into consideration. The ERCD algebra is a pure matrix algebra, i.e. the algebra without any derivatives from the space variables, transformations of reflections, inversions etc.

2. The 32-dimensional subalgebra $\mathrm{A}_{32}=\mathrm{SO}(6) \oplus \mathrm{i} \gamma_{0} \cdot \mathrm{SO}(6) \oplus \mathrm{i} \gamma_{0}\left(\mathrm{i} \gamma_{0}=\hat{\varepsilon}\right)$ of the ERCD algebra is proved to be new and maximal pure matrix algebra of invariance of the $\mathrm{FW}$ equation. Its image $V^{-1}\left(\mathrm{~A}_{32}\right) V$ is the algebra of invariance of the standard Dirac equation in PD-representation.

3. It is shown that some subsets of generators from the $\mathrm{A}_{32}$ have the meaning of the well-known fermionic SL(2,C)- and SU(2)-spins (see formulae (42), (43)), and the others (41) have the meaning of new bosonic SL(2,C)- and SU(2)-spins. Therefore, as the symmetry operators, they have a beneficial physical interpretation.

4. On the basis of the above (see item 3) spins, operators $\vec{x}$ and $\nabla$ (together with well-known fermionic $\mathcal{P}^{\mathrm{F}}$-representation), a new (hidden) bosonic $\mathcal{P}^{\mathrm{B}}$-representation of the proper ortochronous Poincaré group $\mathcal{P}$, as the group of invariance of the Dirac and FW equations, has been found. Using the Bargmann-Wigner analysis this new $\mathcal{P}$-representation has been proved to be the $\mathcal{P}^{\mathrm{B}}$-representation for the Dirac field as the field of particles with the spin 1 .

Therefore, here the assertion that "the Dirac equation can describe the bosons, not only the fermions" is put into consideration as an interesting new possibility. The details and consequences of this assertion will be presented in our further publication, where all results of our papers [12 17] on the $m=0$ case will be repeated for the general case of nonzero mass. Thus, we suggest here a beginning of a natural approach to the supersymmetry of the Dirac equation, in which the Fermi and Bose superpartners are linked by the transformation (44).

The possibilities of applying the ERCD algebra are much more extended than a few examples with the Dirac and FW equations considered above. In general, the ERCD algebra can be applied to any problem, in which the standard CD algebra can be used. Therefore, the introduction of the ERCD algebra is a meaningful independent result in the field of mathematical physics. 


\title{
References
}

1. Darwin C.G., Proc. Roy. Soc. Lon. A, 1928, 118, 654.

2. Mignani R., Recami E., Badlo M., Lett. Nuov. Cim. L, 1974, 11, 572.

3. Laporte O., Uhlenbeck G.E., Phys. Rev., 1931, 37, 1380.

4. Oppenheimer J.R., Phys. Rev., 1931, 38, 725.

5. Good R.H., Phys. Rev., 1957, 105, 1914.

6. Lomont J.S., Phys. Rev., 1958, 111, 1710.

7. Moses H.E., Phys. Rev., 1959, 113, 1670.

8. Daviau C., Ann. Found. L. de Brogl., 1989, 14, 273.

9. Campolattaro A., Intern. Journ. Theor. Phys., 1990, 29, 141.

10. Sallhofer H., Z. Naturforsch. A, 1990, 45, 1361.

11. Keller J. On the electron theory. - In: Proc. of the International Conference "The theory of electron". Mexico, 24-27 September 1995 [Adv. Appl. Cliff. Alg., 1997, 7 (special), 3].

12. Krivsky I.Yu., Simulik V.M., Theor. Math. Phys., 1992, 90, 388.

13. Simulik V.M., Krivsky I.Yu., Adv. Appl. Cliff. Alg., 1998, 8, 69.

14. Simulik V.M., Krivsky I.Yu., Ann. Found. L. de Brogl., 2002, 27, 303.

15. Simulik V.M., Krivsky I.Yu., Rep. Math. Phys. 2002, 50, 315.

16. Simulik V.M. The electron as a system of classical electromagnetic and scalar fields. - In: What is the Electron? Edited by Simulik V.M. Apeiron, Montreal, 2005, 109-134.

17. Simulik V.M., Z. Naturforsch. A, 1994, 49, 1074.

18. Foldy L., Wouthuysen S., Phys. Rev., 1950, 78, 29.

19. Simulik V.M., Krivsky I.Yu. Preprint arXiv: math-ph/0908.3106, 2009.

20. Simulik V.M., Krivsky I.Yu., Rep. Nat. Acad. Sci. Ukraine, 2010, 5, 82.

21. Bogolyubov N.N., Logunov A.A., Oksak A.I., Todorov I.T., The General Principles of the Quantum Field Theory. Nauka, Moskow, 1987 (in Russian).

22. Ibragimov N.H. Groups of Transformations in Mathematical Physics. Nauka, Moskow, 1983, (in Russian).

23. Thaller B., The Dirac Equation. Springer, Berlin, 1992.

24. Foldy L., Phys. Rev., 1956, 102, 568.

25. Gursey F., Nuovo Cim., 1958, 7, 55.

26. Ibragimov N.H., Theor. Math. Phys., 1969, 1, 350.

\section{Фермі-Бозе дуалізм рівняння Дірака та розширена дійсна алгебра Кліффорда-Дірака}

\author{
І.Ю. Кривський, В.М. Симулик \\ Інститут електронної фізики, Національна академія наук України, \\ вул. Університетська, 21, 88000 Ужгород
}

На основі симетрійного аналізу стандартного рівняння Дірака з ненульовою масою доведено, що це рівняння може описувати не лише ферміони зі спіном 1/2, але й бозони зі спіном 1. Знайдено нові бозонні симетрії рівняння Дірака як у представленні Фолді-Вотхойзена, так і у представленні Паулі-Дірака. Серед цих симетрій (поряд з 32-вимірною чисто матричною алгеброю інваріантності) доведено нову, фізично важливу симетрію Пуанкаре спіна 1 згаданого рівняння. Для виконання зазначених доведень введено в розгляд 64-вимірну розширену дійсну алгебру Кліффорда-Дірака.

Ключові слова: спінорне поле, симетрія, теоретико-груповий аналіз, суперсиметрія, представлення Фолді-Вотхойзена, алгебра Кліффорда-Дірака 
